\title{
Avaliação do Medo de Queda, Confiança no Equilíbrio, Marcha e Equilíbrio Corporal de Idosas Praticantes Regulares do Método Pilates
}

\section{Evaluation of Fear of Falling, Confidence in Balance, Gait and Body Balance of Seniors Regular Pilates Method Practitioners}

\author{
Marcelo de Maio Nascimento ${ }^{1}$ \\ Cássia Poliana Príncipe Nunes ${ }^{2}$ \\ Eddie Nara Dantas Passos Rosa ${ }^{3}$ \\ Deborah Silva de Menezes ${ }^{3}$ \\ Ana Carolina Pereira Eugênio ${ }^{3}$ \\ Paloma Sthefane Teles Silva ${ }^{3}$ \\ Lucas Vinícius Ferreira Vieira de Oliveira ${ }^{4}$
}

\section{RESUMO}

Objetivo: Avaliar o desempenho de mulheres idosas praticantes regulares do método Pilates em testes de marcha e equilíbrio corporal, assim como, a percepção do medo de queda e da confiança no equilíbrio, além de estimar o risco de queda da população avaliada. Metodologia: Trata-se de um estudo do tipo transversal observacional descritivo, desenvolvido com 254 mulheres $(67,35 \pm 5,20$ anos), praticantes regulares do método Pilates, com e sem histórico de quedas. Foram utilizados os seguintes instrumentos: Questionário (sociodemográfico, comorbidades, medicamentos e histórico de quedas), Mini-exame do estado mental de saúde (MEEM), Falls efficacy scale (FES), Balance confidence scale (ABC), Teste de equilíbrio corporal (TEC), Dynamic gait index (DGI), Time up and go (TUG), incluindo sua versão motora (TUGm) e cognitiva (TUGc). Resultados: Sexagenárias e septuagenárias mostraram desempenho satisfatório na avaliação da marcha na condição simples TUG $(p<0,01)$, de dupla tarefa TUGm $(p<0,01)$ e TUGc $(p<0,01)$ e funcional $D G I(p<0,01)$. O TEC classificou o desempenho do equilíbrio de sexagenárias como bom $(p<0,01)$ e fraco para septuagenárias, entre $75-70$ anos $(p<0,01)$. Não foi verificado medo de queda na população avaliada $(p \geq 0,05)$. Idosas $\geq 75$ anos indicaram maior confiança no equilíbrio $(p \geq 0,05)$, sugerindo comportamento menos atento para os riscos de queda. $O$ modelo da análise de regressão linear múltipla foi significativo $\left[F(1,71)=5,358 ; p=0,024 ; R^{2}=0,449\right]$, as variáveis labirintite e confiança no equilíbrio se mostraram previsoras do risco de queda em $86 \%$ e $55 \%$, respectivamente. Conclusão: A prática regular do Pilates mostrou ser eficaz à prevenção do risco de queda de mulheres sexagenárias e septuagenárias.

\section{DESCRITORES}

Quedas. Medo de Cair. Envelhecimento. Pilates.

\begin{abstract}
Objective: To evaluate the performance of elderly women who regularly practice the Pilates method in gait and body balance tests, as well as the perception of fear of falling and confidence in balance, in addition to estimating the risk of falling of the assessed population. Methodology: This is a descriptive observational cross-sectional study, developed with 254 women $(67.35$ \pm 5.20 years), regular Pilates practitioners, with and without a history of falls. Mental Health Status Examination (MMSE), Falls Efficacy Scale (FES), Balance Confidence Scale (ABC), Body Balance Test (TEC), Dynamic Gait Index (DGI), Time Up and Go (TUG), including its motor (TUGm) and cognitive (TUGc) versions. Results: Sexagenarians and septuagenarians showed satisfactory performance in gait assessment in the simple condition $(p<0.01)$ and TUGc $(p<0.01)$ and functional DGI tasks $(p<0.01)$. The TEC classified the performance of the sexagenarian balance as good $(p<0.01)$ and weak for the septuagenarians, between $75-70$ years $(p<0.01)$. There was no fear of falling in the evaluated population $(p \geq 0.05)$. Elderly $\geq 75$ years indicated greater confidence in the balance $(p \geq 0.05)$, suggesting less attentive behavior for the risks of falling. The model of multiple linear regression analysis was significant $[F$ $\left.(1,71)=5,358 ; p=0.024 ; R^{2}=0.449\right]$, the variables Labyrinthitis and Confidence in Balance were shown to predict the risk of falling by $86 \%$ and $55 \%$, respectively. Conclusion: The regular practice of Pilates has been shown to be effective in preventing the risk of falling of sexagenarian and septuagenarian women.
\end{abstract}

\section{DESCRIPTORS}

Falls. Fear of Falling. Aging. Pilates.

${ }^{1}$ Doutor em Ciências do Movimento Humano Colegiado de Educação Física da Universidade Federal do Vale do São Francisco (UNIVASF).

${ }^{2}$ Psicóloga formada pela UNIVASF. Residente no Instituto de Medicina Integral Professor Fernando Figueira-IMIP, Recife/PE.

${ }^{3}$ Psicóloga formado pela UNIVASF. Psicóloga no Município de Juazeiro-BA.

${ }_{4}^{4}$ Professor de Educação Física formado pela UNIVASF. Professor de Educação Física em Petrolina-PE. 
e acordo com estimativas do Instituto Brasileiro de Geografia e Estatística $\left(\right.$ IBGE $^{1}$, no ano de 2050, a população brasileira, em idade igual ou superior a 60 anos corresponderá a 27,8 milhões de habitantes. Como responsável pelo fato, admite-se o aumento da expectativa média de vida, que hoje, no Brasil, é de 74 anos, a baixa dos índices de natalidade e os avanços na área da saúde ${ }^{1}$. Isso significa dizer que o Brasil apresentará um número considerável de septuagenários e octogenários, uma população que tendencialmente exibe prevalência de doenças e dependências funcionais. O caso justifica a realização de investigações que ampliem e qualifiquem o entendimento sobre as particularidades do envelhecimento humano.

O envelhecimento humano é considerado um processo responsável por alterações da caráter biopsicofuncional, sobrevindos de uma série de mudanças graduais na composição molecular e celular do organismo ${ }^{2}$. O processo provoca, entre outros, a baixa do desempenho funcional, dificultando a adaptação do indivíduo ao meio, o que reduz a autonomia. Dentre as alterações funcionais existe o déficit do equilíbrio corporal e a redução da velocidade da marcha ${ }^{3,4}$. O equilíbrio incide em uma capacidade motora fundamental à sustentação do centro de gravidade na posição estática sobre os limites da base de apoio do corpo, com um mínimo de oscilação corporal ${ }^{5}$. A ação é complexa, visto que, inicialmente, informações sobre a postura são captadas pelo sistema visual, vestibular e somatossensorial e enviadas ao sistema nervoso central (SNC) encarregado de processar os dados e informar o aparelho muscular sobre ajustes posturais exigidos ${ }^{6}$.
Alterações nos sistemas sensoriais aumentam o risco de quedas ${ }^{3}$. As quedas são responsáveis por lesões, dias de hospitalização, impossibilitando a pessoa, em certos casos, de realizar suas atividades de vida diária (AVD), como também, afetam negativamente a qualidade de vida (QV) tanto da pessoa que caiu, como dos familiares. Nesse contexto, a avaliação do risco de queda de idosos incide em questão de saúde pública. ${ }^{8}$ destacaram que os procedimentos também devem considerar o medo de cair e a confiança no equilíbrio, pois, a insegurança acaba conduzindo a pessoa, muitas vezes, a novas quedas. Conforme os autores, idosos caidores relatam o medo de cair como uma questão traumática, indicando que o evento é uma experiência emocional negativa ${ }^{7}$. O medo de cair afeta a confiança no equilíbrio, pois inibe a pessoa de elaborar estratégias para evitar a queda, não superando desafios ${ }^{9}$. Acredita-se que idosos com essa característica foquem sua atenção, tendencialmente, sobre as próprias limitações, intensificando o medo de cair ${ }^{10}$.

Em estudo realizado com 1.088 idosos de uma comunidade (65-89 anos), Kumar e colaboradores ${ }^{10}$, constataram uma forte associação entre o medo de queda com os fatores de ordem física e psicológica. Este tipo de medo pode se transformar em um sintoma depressivo, reduzindo gradativamente a mobilidade do idoso. Segundo labone et al. ${ }^{11}$, ao desenvolverem um programa de prevenção de quedas com 69 idosos (77,8 $\pm 8,9$ anos) portadores de transtornos depressivos, foi verificado que após 12 semanas de intervenção existiu melhora do equilíbrio e da marcha, bem como, uma associação entre os transtornos depressivos e o medo de cair. Além 
disso, sabe-se que idosos com histórico de quedas se tornam, em geral, mais cautelosos, diminuindo a velocidade e a amplitude das passadas, o que aumenta a exposição para novas eventos ${ }^{12}$.

Pimentel e Scheicher ${ }^{13}$ avaliaram sexagenárias fisicamente ativas identificando o medo de cair tanto em indivíduos com, como sem histórico de queda. A literatura também afirma que a prevalência do medo de cair é maior em indivíduos do sexo feminino, existindo grande relação com o avanço da idade $^{14}$. Em uma investigação desenvolvida com 147 idosos (60-92 anos) residentes em Diamantina-MG, os autores verificaram níveis de correlação significativa entre o medo de queda e o desempenho em testes de marcha e equilíbrio ${ }^{12,14}$. Os achados corroboraram com a literatura especializada, que afirma que alterações no padrão da marcha incide em fator associado ao risco de queda ${ }^{15}$, ou seja, idosos lentos estão mais propensos a cair.

Uma medida sugerida à prevenção de quedas de indivíduos idosos consiste na prática regular de exercícios físicos ${ }^{16,17}$, dentre eles, existe o método Pilates. Estudos de revisão sistemática ${ }^{18}$ e meta-análise ${ }^{19}$ realçaram seus benefícios para manter e/ou desenvolver o equilíbrio corporal, o padrão da marcha, a força, a flexibilidade, a consciência corporal, prevenindo quedas ${ }^{20-22}$. A prática regular do Pilates também é capaz de elevar a confiança no equilíbrio, reduzindo o medo do idoso de cair $^{23}$, além de favorecer a percepção da QV e do bem-estar ${ }^{24}$.

Diante do exposto, o presente estudo teve como objetivo avaliar o desempenho de mulheres idosas praticantes regulares do método Pilates em testes de marcha e de equilíbrio, a percepção do medo de queda e da confiança no equilíbrio, além de estimar o risco de queda dessa população.

\section{METODOLOGIA}

Trata-se de um estudo transversal, observacional e descritivo ${ }^{25}$. As participantes foram recrutadas por meio de convite pessoal durante às aluas do programa de extensão "Pilates e o Idoso", oferecido pelo curso de Educação Física da Universidade Federal do Vale do São Francisco (UNIVASF). Neste programa, a prática do Pilates é realizada duas vezes por semana (60 minutos). Para verificar se o número de avaliações alcançado apresentava poder estatístico foi realizado cálculo amostral a posteriori pelo softwear $\mathrm{G}^{*} \mathrm{Power}$ 3 , tendo como base: 1) desenho do estudo; 2) taxa de erro de tipo I de $5 \%(\alpha=0,05) ; 3)$ tamanho de efeito de 0,20 ; e 4) 254 integrantes do sexo feminino, obtendo-se poder estatístico de $80 \%(1-\beta=0,80)$. As participantes foram estratificadas em faixas etárias de cinco em cinco anos: G1 (60-64 anos): $n=84, \mathrm{G} 2$ (65-69 anos): $n=93$, G3 (70-74 anos): $n=45$ e G4 (75-79 anos): $n=31$. Como critério de inclusão foi adotado idade $\geq 60$ anos, prática do Pilates com tempo mínimo de três meses e frequência de $75 \%$ nas atividades. Foram excluídas aquelas que apresentaram lesão muscular, articular ou óssea no período da avaliação, igualmente, doenças neurológicas como doença de Parkinson ou acidente vascular encefálico, não completaram todas as etapas da investigação ou não assinaram o Termo de Consentimento Livre e Esclarecido (TCLE). A coleta dos dados ocorreu entre novembro de 2017 e janeiro de 2018. O estudo 
foi aprovado pelo Comitê de Ética em Pesquisa com Seres Humanos da Universidade Federal do Vale do São Francisco/UNIVASF (CAAE: 44113715.3.0000.5196).

\section{Procedimentos}

A investigação compreendeu quatro etapas: Fase 1) Aplicação de um questionário para coleta de dados sociodemográficos (idade, escolaridade), informações sobre comorbidades e a incidência de quedas nos últimos 12 meses.

Fase 2) Mini-exame do estado mental de saúde (MEEM): O MEEM avalia a função cognitiva da pessoa, permitindo o rastreamento da demência a partir de sete domínios. Para as idosas analfabetas foi considerado o escore mínimo de 13 pontos, para aquelas com um a quatro anos de escolaridade, 18 pontos e 26 pontos, para as idosas com escolaridade de cinco anos ou mais ${ }^{26}$.

Fase 3) Dados antropométricos: A massa corporal e a estatura foram determinadas por meio de uma balança mecânica, até $300 \mathrm{Kg}$ (Welmy, Brasil), com régua antropométrica de escala até $2 \mathrm{~m}$. O índice de massa corporal (IMC) foi estabelecido mediante a fórmula: massa $(\mathrm{Kg})$ /estatura $\left(\mathrm{m}^{2}\right)$. A avaliação do estado nutricional foi estabelecida segundo os critérios da $\mathrm{OMS}^{27}$.

Fase 4) Exame do medo de quedas (FES) e confiança no equilíbrio (ABC):

a) A Falls Efficacy Scale (FES) ${ }^{28}$ conhecida como escala para avaliação da autoeficácia relacionada às quedas. Ela realiza uma estimativa sobre o medo de queda a partir de 16 perguntas, investigando a preocupação do indivíduo quanto à possibilidade de cair durante a realização de tarefas específicas (em ambiente doméstico ou ao ar livre). O escore total pode variar de 16 (ausência de preocupação) a 64 (preocupação extrema). No presente estudo, admitiu-se uma pontuação <23 pontos para maior independência e $\geq 23$ pontos para associação com medo de queda ${ }^{28}$.

b) Activities-specific Balance Confidence Scale $(\mathrm{ABC})$ foi apresentada por Marques et al ${ }^{9}$. Ela busca, por meio de entrevista, averiguar o nível de confiança do indivíduo para não perder o equilíbrio ou ficar instável. A ABC é composta por 16 perguntas, com ênfase em atitudes da pessoa durante o desempenho das AVD. A sua pontuação é realizada por meio da porcentagem atribuída pelo entrevistado sobre seu nível de confiança para cada AVD. Os valores variam entre $0 \%$ (sem confiança) a $100 \%$ (confiança completa), com resultado total entre 0 (mínimo) e 1600 (máximo). O valor é dividido por 16 , resultando na avaliação final ${ }^{9}$. Fase 5) Avaliação do risco de quedas pelos testes DGI, TEC, TUG, TUGm e TUGc):

a) Dynamic Gait Index (DGI)/Índice de Marcha Dinâmica: A escala tem por fim avaliar a marcha em diferentes situação (superfície plana, variação da velocidade, rotações da cabeça), predizendo a probabilidade de que$\mathrm{da}^{29}$. Possui oito tarefas funcionais. $\mathrm{O}$ teste apresenta escore máximo de 24 pontos, cada tarefa recebe de zero a três pontos. Em idosos ( $\geq 60$ anos), o escore igual ou inferior a 19 pontos prediz o risco de queda, enquanto que a marcha segura é determinada por valores superiores a 22 pontos $^{29}$.

b) Teste de Equilíbrio Corporal (TEC): Possui 14 tarefas, sete avaliam o equilíbrio estático e sete o equilíbrio 
dinâmico. Dessas, seis avaliam a regulação exteroceptiva (olhos abertos) do equilíbrio estático e dinâmico, oito a regulação interoceptiva (olhos fechados) $)^{30}$. Seus itens possuem ordem crescente de dificuldade, a pontuação é dicotômica, atribuindo-se zero para o objetivo não atingido e um para o atingido. A interpretação dos resultados segue um sistema de normativas de êxito, categorizadas por faixas etárias e gênero ${ }^{30}$.

c) Timed Up and Go (TUG): O teste TUG é utilizado junto à avaliação da mobilidade e do equilíbrio funcional, requisitando o autocontrole postural. Sentado em uma cadeira de $45 \mathrm{~cm}$, com apoio para os braços, o avaliado deve se erguer e caminhar três (3) metros, realizar um giro de $180^{\circ} \mathrm{em}$ um cone, retornar até a cadeira e se sentar ${ }^{15}$. Considerou-se para adultos independentes a realização do teste em até $10 \mathrm{seg}$, como normal (sem risco de quedas), resultados entre 11-20 seg, indicam a independência parcial (baixo risco de quedas) e valores acima de $20 \mathrm{seg}$, apontam para o déficit da mobilidade física (alto risco de quedas) $)^{15}$.

d) TUG motor (TUGm): O teste possui as mesmas diretrizes do TUG convencional, porém, ocorre o transporte, com ambas as mãos, de uma bandeja de papelão com $25 \mathrm{~cm}$ de raio, que possui um copo de plástico vazio com $12 \mathrm{~cm}$ de altura. A tarefa consiste no transporte da bandeja sem que o copo caia. A pontuação considera os valores normativos do TUG simples ${ }^{15}$.

e) TUG cognitivo (TUGc): O avaliado executa o TUG convencional, entretanto, durante o percurso conta em ordem decrescente e em voz alta, iniciando no número cem. Para a pontuação foram considerados os valores normativos do TUG simples ${ }^{15}$.

A normalidade dos dados foi verificada por meio do teste de Kolgomorov-Smirnov, verificando-se distribuição normal. A estatística descritiva (média, desvio-padrão e frequência) foi utilizada para apresentação dos resultados. A verificação das variáveis categóricas (queda nos últimos 12 meses e comorbidades) foi realizada pelo teste do Qui-Quadrado, corrigidas pelo Exato de Fischer. O teste da Anova foi aplicado para verificar os níveis de significância das várias (ABC e FES, TUG, TUGm, TUGc, GDI, TEC), consideradas como previsoras do risco de queda. As comparações entre os grupos foi processada pelo post hoc de Bonferroni. Diferenças estatísticas entre pares foram calculadas pelo teste $T$ Student de amostras independentes. Por fim, considerando que o risco de queda não pode ser explicado por um único agente causal foi executada a análise de regressão linear múltipla. Considerou-se como varável dependente do modelo a resposta sobre quedas nos últimos 12 meses, por conseguinte, integraram o cálculo apenas as variáveis independentes que mostraram níveis de significância no modelo univariado (audição, labirintite, escala ABC, os testes TUG, TUGm, TUGc, DGI, TEC). A ordem adotada para inserção das variáveis foi do maior para o menor (modelo forward), respeitando a magnitude do coeficiente de 
correlação de Spearman. Razões de chances (OR, IC 95\%) foram utilizadas para apresentação dos resultados. Os dados foram tabulados e processados no programa estatístico SPSS para Windows ${ }^{\circledR}$ versão 21.0 . Os efeitos foram testados no nível de 0,05 de significância.

\section{RESULTADOS}

A Tabela 1 apresenta as principais características das participantes do estudo. A idade média da população estuda foi de $67,35 \pm 5,20$ anos. De acordo com o exame do MEEM não foram identificados casos de demência $(p \geq 0,05)$. Diferença estatística foi verificada para a massa corporal $(p \leq 0,05)$. Em relação às comorbidades, o déficit de audição e a labirintite mostraram resultados significativos $(p \leq 0,05)$. O maior consumo de medicamentos foi constatado entre as septuagenárias, contudo sem indicação de interação medicamentosa $(p \geq 0,05)$. Segundo o autorrelato de quedas para os últimos 12 meses, faixas entre 60-64 anos e 75-79 indicaram os maiores escores, com índices de 30,1\% e $31,0 \%$, respectivamente $(p \geq 0,05)$.

$\mathrm{Na}$ Tabela 2 são apresentados os resultados para os instrumentos aplicados. Com respeito ao medo de quedas (FES), todos os grupos mostraram escores semelhantes, sem indicação sobre medo de queda $(p \geq 0,05)$. Idosas do G1 e G3 apresentaram maior preocupação com o equilíbrio ( $A B C)$, enquanto que idosas longevas (75-79) indicaram maior

Tabela 1. Principais características dos participantes do estudo

\begin{tabular}{|c|c|c|c|c|c|}
\hline Variáveis & $\begin{array}{c}\text { 60-64 anos } \\
(n=84) \\
\text { Média/DP }\end{array}$ & $\begin{array}{c}\text { 65-69 anos } \\
(\mathrm{n}=93) \\
\text { Média/DP }\end{array}$ & $\begin{array}{c}\text { 70-74 anos } \\
(n=45) \\
\text { Média/DP }\end{array}$ & $\begin{array}{c}\text { 75-79 anos } \\
(n=31) \\
\text { Média/DP }\end{array}$ & $p$ \\
\hline Idade & $61,85 \pm 1,87$ & $66,99 \pm 1,41$ & $71,80 \pm 1,35$ & $76,45 \pm 1,45$ & $0,001^{*}$ \\
\hline Escolaridade Anos) & $8,82 \pm 2,82$ & $7,52 \pm 2,66$ & $6,45 \pm 3,28$ & $5,77 \pm 1,82$ & 0,184 \\
\hline MEEM & $26,71 \pm 2,61$ & $26,37 \pm 3,71$ & $26,19 \pm 3,59$ & $23,63 \pm 5,05$ & 0,110 \\
\hline Massa $(\mathrm{kg})$ & $87,67 \pm 38,70$ & $80,11 \pm 30,94$ & $79,32 \pm 3,83$ & $58,82 \pm 9,79$ & $0,015^{*}$ \\
\hline Estatura $(\mathrm{cm})$ & $1,53 \pm 6,60$ & $1,51 \pm 6,32$ & $1,54 \pm 7,14$ & $1,50 \pm 3,22$ & 0,116 \\
\hline IMC $\left(\mathrm{kg} / \mathrm{m}^{2}\right)$ & $28,35 \pm 7,32$ & $28,42 \pm 5,15$ & $28,21 \pm 6,61$ & $26,28 \pm 3,07$ & 0,850 \\
\hline Medicamentos & $2,69 \pm 1,55$ & $2,91 \pm 2,02$ & $3,43 \pm 2,17$ & $3,15 \pm 1,07$ & 0,302 \\
\hline EF (meses) & $22,4 \pm 2,4$ & $23,6 \pm 3,6$ & $18,2 \pm 5,8$ & $19,3 \pm 4,7$ & \multirow{6}{*}{0,245} \\
\hline Quedas & \multicolumn{4}{|c|}{ n (\%) } & \\
\hline Sim & $25(30,1)$ & $17(18,7)$ & $09(20,5)$ & $9(31,0)$ & \\
\hline Não & \multirow[t]{3}{*}{$58(69,9)$} & \multirow[t]{3}{*}{$34(81,3)$} & \multirow[t]{3}{*}{$35(79,5)$} & \multirow[t]{3}{*}{$20(69,0)$} & \\
\hline Comorbidades & & & & & \\
\hline Audição & & & & & \\
\hline Sim & $9(12,9)$ & $11(13,6)$ & $7(17,5)$ & $10(41,7)$ & \multirow[t]{3}{*}{$0,008^{\dagger}$} \\
\hline Não & \multirow[t]{2}{*}{$61(87,1)$} & \multirow[t]{2}{*}{$70(86,4)$} & \multirow[t]{2}{*}{$33(82,5)$} & \multirow[t]{2}{*}{$14(58,3)$} & \\
\hline Visão & & & & & \\
\hline Sim & \multirow{3}{*}{$\begin{array}{l}56(80,0) \\
14(20,0)\end{array}$} & \multirow{3}{*}{$\begin{array}{l}67(82,7) \\
14(17,3)\end{array}$} & \multirow{3}{*}{$\begin{array}{c}33(82,5) \\
7(17,5)\end{array}$} & \multirow{3}{*}{$\begin{array}{c}18(75,0) \\
6(25,0)\end{array}$} & \multirow[t]{3}{*}{0,845} \\
\hline Não & & & & & \\
\hline Labirintite & & & & & \\
\hline Sim & $14(20,0)$ & $22(27,2)$ & $12(30,0)$ & $7(29,2)$ & \multirow{2}{*}{$0,018^{t}$} \\
\hline Não & $56(80,0)$ & $59(72,8)$ & $28(70,0)$ & $17(70,8)$ & \\
\hline
\end{tabular}

$\mathrm{DP}=$ Desvio padrão, MEEM=Mini Exame do Estado de Saúde Mental, $\mathrm{kg}=$ quilogramas, $\mathrm{cm}=$ centímetros, $\mathrm{m}^{2}=$ metro quadrado, $\mathrm{EF}=$ exercício físico, ${ }^{*} p \leq 0,050=$ ANOVA, ${ }^{\prime} p \leq 0,050=$ Chi-Quadrado. 
confiança no equilíbrio $(p \geq 0,05)$. A avaliação da marcha na situação de tarefa simples (TUG) e dupla (TUGm e TUGc) destacou baixa do desempenho com o avanço da idade $(p \leq 0,05)$. Nessa perspectiva, septuagenárias mostraram maior lentidão que sexagenárias. Os resultados do DGI não indicaram comprometimentos de ordem funcional $(p \leq 0,05)$. Seguindo a ordem dos resultados, o teste TEC indicou desempenho do equilíbrio estático e dinâmico inversamente proporcional à idade $(p \leq 0,05)$, com leve risco de queda para septuagenárias, entre 75-79 anos.

A Tabela 3 apresenta os resultados da análise de regressão linear múltipla, desenvolvida para estimar o risco de queda sob o efeito de comorbidades, medo de quedas, confiança no equilíbrio e o desempenho nos testes de marcha e do equilíbrio corporal. O modelo obtido foi estatisticamente significativo $\left[F(1,71)=5,358 ; p=0,024 ; R^{2}=0,449\right]$, apontando a labirintite $(\beta=0,135 ; t=2,071 ; p \leq 0,05)$ e a confiança no equilíbrio $(\beta=0,012 ; t=2,563$; $\mathrm{p} \leq 0,05)$ como previsores do risco de queda da população avaliada.

\section{DISCUSSÃO}

O principal achado do presente estudo foi que a labirintite e a confiança no equilíbrio mostraram aumentar o risco de queda em $86 \%$ e 55\%, respectivamente. Uma explicação para

Tabela 2. Resultados das escalas de medo e confiança do equilíbrio, testes de marcha e equilíbrio corporal

\begin{tabular}{|c|c|c|c|c|c|}
\hline Variáveis & $\begin{array}{c}\text { 60-64 anos } \\
(n=84) \\
\text { Média/DP }\end{array}$ & $\begin{array}{c}\text { 65-69 anos } \\
(n=93) \\
\text { Média/DP }\end{array}$ & $\begin{array}{c}\text { 70-74 anos } \\
(\mathrm{n}=45) \\
\text { Média/DP }\end{array}$ & $\begin{array}{c}\text { 75-79 anos } \\
(n=31) \\
\text { Média/DP }\end{array}$ & $p$ \\
\hline FES & $27,39 \pm 7,27$ & $26,71 \pm 8,29$ & $23,75 \pm 8,68$ & $28,33 \pm 10,71$ & 0,354 \\
\hline $\begin{array}{l}\text { ABC } \\
\text { Marcha }\end{array}$ & $52,46 \pm 41,21$ & $60,83 \pm 34,92$ & $59,74 \pm 37,48$ & $80,71 \pm 15,30$ & $0,038^{*}$ \\
\hline TUG & $9,34 \pm 1,70^{\mathrm{a}, \mathrm{b}}$ & $9,22 \pm 1,52^{c}$ & $10,41 \pm 2,08^{\mathrm{a}, \mathrm{c}}$ & $10,54 \pm 2,29^{b}$ & $<0,001^{*}$ \\
\hline TUGm & $9,81 \pm 1,77^{a, b}$ & $9,81 \pm 1,90^{\mathrm{c}}$ & $10,77 \pm 2,20^{\mathrm{a}, \mathrm{c}}$ & $10,97 \pm 2,01^{b}$ & $0,007^{*}$ \\
\hline TUGc & $10,72 \pm 2,93^{a}$ & $10,66 \pm 2,55^{c}$ & $11,87 \pm 3,52^{c}$ & $12,26 \pm 3,56^{a}$ & $0,037^{*}$ \\
\hline Equilíbrio & & & & & \\
\hline DGI & $21,42 \pm 2,44^{a}$ & $21,57 \pm 2,00$ & $20,42 \pm 4,03$ & $20,16 \pm 2,11^{a}$ & $0,035^{*}$ \\
\hline TEC & $6,38 \pm 3,33^{a, b}$ & $5,50 \pm 3,33$ & $4,71 \pm 3,23^{a}$ & $3,80 \pm 2,18^{b}$ & $0,004^{*}$ \\
\hline
\end{tabular}

$\mathrm{DP}=$ Desvio padrão, $\mathrm{FES}=E s c a l a$ de medo de quedas, $\mathrm{ABC}=$ Escala de confiança no equilíbrio durante atividade específica, TUG=Timed Up and Go, TUGm=TUGmotor, TUGc=TUGcognitivo, DGI=Dynamic Gait Index, TEC=Teste de Equilíbrio Corporal, " $p<0,050=A N O V A$, a,b,c $p<0,050=$ Mann Whitney.

Tabela 3. Modelo de regressão ajustado para comorbidades, medo de quedas, confiança no equilíbrio, desempenho da marcha e equilíbrio corporal

\begin{tabular}{lcccc}
\hline \multicolumn{1}{c}{ Variáveis } & $\mathrm{b}$ & Erro Padrão & IC 95\% & $\mathrm{p}$ \\
\hline Labirintite & 0,135 & 0,065 & $0,140(0,006-1,263)$ & $0,042^{*}$ \\
\hline $\mathrm{ABC}$ & 0,012 & 0,005 & $0,450(0,002-1,023)$ & $0,044^{*}$ \\
\hline
\end{tabular}

Legenda: $\mathrm{ABC}=$ Confiança no Equilíbrio; ${ }^{*} \mathrm{p} \leq 0,05$. 
o fato é que problemas no labirinto potencializam a tontura, a sensibilidade auditiva, influenciando a regulação do equilíbrio corporal. Por conseguinte, alterações no desempenho da aparelho auditivo dificultam o desempenho da marcha em linha reta, bem como, a associação da marcha com mudanças da direção do olhar, como para cima-baixo e para os lados $^{6}$. Isso sobrevém de problemas no vestíbulo ou na cóclea, que impedem o SNC de receber informações relativas à posição do corpo no espaço ${ }^{6}$. Diante disso, indivíduos com labirintite, quando questionados sobre a confiança no equilíbrio, em uma escala de 0-100\%, responderam não se sentiram aptos para atribuir altos escores, indicando baixa segurança sobre a condição do equilíbrio ${ }^{9,18}$.

De acordo com a análise estatística, a população avaliada não era caidora, visto que $23,7 \%$ das participantes relataram quedas nos últimos 12 meses. Dessas, 70,0\% eram sexagenárias e 30\% septuagenárias. Com relação ao exame do medo de quedas, comparativamente, a escala FES mostrou para todas as faixas etárias baixos níveis de preocupação com o equilíbrio ( $p \geq 0,05)$. Por outro lado, em se tratando da confiança no equilíbrio, a escala $A B C$, idosas entre 75-79 anos indicaram alta confiança no equilíbrio: valores entre $80 \%-100 \%$. Enquanto, os membros dos demais grupos exibiram resultados entre $52 \%-60 \%(p \geq 0,05)$. A literatura especializada salienta que uma alta confiança no equilíbrio, seguida pelo baixo medo de queda, como foi constatado no grupo entre 75 a 79 anos, mostra negligência do risco de cair, aumentando o risco de queda. No caso das idosas dos demais grupos, os resultados nas escalas FES e $A B C$ podem ser interpretados como de indivíduos com perfil cauteloso em relação à queda.

No presente estudo, como esperado, verificou-se relação inversamente proporcional entre o desempenho da marcha e o avanço da idade ${ }^{3}$. Entretanto os resultados não classificaram a população avaliada com risco de queda ${ }^{15}$. Estudos de revisão sistemática e meta-análise mostraram que tempos $\geq 12$ segundos em testes de marcha servem como medida funcional à determinação de risco para queda ${ }^{12,7}$. Uma explicação para o bom desempenho da marcha, entre sexagenárias e septuagenárias pode ser atribuída à prática regular do Pilates. Estudos anteriores, destacaram existir aumento significativo da velocidade da marcha entre idosos praticantes do Pilates, após cinco semanas de treinamento ${ }^{21,20}$, o que torna o método uma medida eficaz na prevenção de quedas ${ }^{18,19}$.

Sabe-se que o envelhecimento causa ao organismo uma série de alterações de ordem fisiológica, responsáveis por distúrbios no funcionamento do sistema sensorial (visão, audição e propriocepção), que é encarregado pela regulação do equilíbrio corporal ${ }^{6,8}$. Conforme a avaliação sensorial do equilíbrio, os escores do teste TEC indicaram um bom desempenho para o equilíbrio estático e dinâmico de sexagenárias e das septuagenárias, com exceção daquelas entre 75-79 anos que indicaram classificação regular para o controle postural $(p \leq 0,05)$. Os achados mostraram claramente que o envelhecimento causa alterações sobre o controle postural.

Conforme Kleiner et al. ${ }^{6}$ distúrbios nos sistemas sensoriais, visual, vestibular e somatossensorial dificultam a emissão de informações posturais ao SNC. Por essa razão, o SNC 
fica impedido de arquitetar representações sobre os segmentos corporais, em relação ao ambiente $^{30}$. Por conseguinte, a emissão dos comandos reguladores da postura corporal ao sistema muscular se tornam lentos, logo, incapazes de realizar os justes posturais necessários $^{6}$. Admite-se que a presente investigação possui limitações. Primeiro, porque a o número de idosas caidores e não caidores não foi homogênea. Segundo, que devido a ausência de um grupo controle formado por idosas sedentárias não foi possível traçar associações de causalidade relativas à eficácia da prática do método Pilates como medida à prevenção de quedas. Por fim, reconhece-se que o delineamento transversal não permite a generalização dos resultados.

\section{CONCLUSÃO}

Os achados desta investigação foram conclusivos de que, entre mulheres idosas, mesmo aquelas fisicamente ativas, distúrbios do sistema vestibular, em especial, a labirintite devem ser considerados como fator respon-

\section{REFERÊNCIAS}

1. IBGE-Instituto Brasileiro de Geografia e Estatística. Projeção da População do Brasil por sexo e idade: 2000-2060.

2. OMS-Organização Mundial de Saúde. Relatório Mundial de Envelhecimento e Saúde. 2015;(1):1-29. Disponível em: http://sbgg.org.br/wp-content/uploads/2015/10/OMS-ENVELHECIMENTO-2015.

3. Palumbo P, Klenk J, Cattelani L, Bandineli S, Ferrucci L, Rapp K et al. Predictive Performance of a Fall Risk Assessment Tool for Community-Dwelling Older People (FRAT-up) in 4 European Cohorts. J Am Med Dir Assoc. 2016;17(12):1106-1113.

4. Auvinet B, Touzard C, Montestruc F, Delafond A, Goeb V. Gait disorders in the elderly and dual task gait analysis: a new approach for identifying motor phenotypes. J Neuroeng Rehabil. 2017;14(1):1-14. sável pelo aumento do risco de queda. Por conseguinte, verificou-se associação entre a labirintite com a diminui da confiança no equilíbrio. Corroborando com estudos anteriores, o desempenho motor em testes de marcha e equilíbrio se apresentou inversamente proporcional ao aumento da idade. Sugere-se a realização de futuras investigações, que comparem idosas praticantes regulares do método Pilates com praticantes de outras modalidades e/ou idosas sedentárias, a fim de que os resultados possam ampliar o desfecho da presente investigação. Ademais, que exista o acompanhamento longitudinal dos idosos, visto que a avaliação constante da marcha, do equilíbrio, o medo de queda e a confiança no equilíbrio servem como marcadores do estado geral de saúde do idoso.

\section{AGRADECIMENTOS}

Os autores agradecem a Pró-Reitoria de Extensão (PROEX) da Universidade Federal do Vale do São Francisco pelo apoio às atividades do projeto Pilates e o Idoso.

5. Shumway-CookA, Woollacott WH. Controle Motor: Teoria E Aplicações Praticas. $2^{a}$ Ed. Barueri: Manole, p. 179-208, 2003.

6. Kleiner AFR, Schlitter DXDC, Sánchez-Arias MDR. O papel dos sistemas visual, vestibular, somatosensorial e auditivo para o controle postural. Rev Neurociencias. 2011;19(2):349-357.

7. Lusardi MM, Middleton A, Wingood M, Criss M, Osborne J. Determining Risk of falls in community dwelling older adults: A systematic review and meta-analysis using posttest probability. J Geriatr Phys Ther. 2017:40(1):1-36.

8. Tinetti ME, Richmand D, Powell L. Falls efficacy as a measure of fear of falling. J Gerontol. 1990;45(6):239-243. 
9. Marques AP, Mendes YC, Taddei U, Pereira CAB, Assumpção A. Brazilian-Portuguese translation and cross cultural adaptation of the activities-specific balance confidence (ABC) scale. Brazilian J Phys Ther. 2013;17(2):170-177.

10. Kumar A, Carpenter H, Morris R, lliffe S, Kendrick D. Which factors are associated with fear of falling in community-dwelling older people? Age Ageing. 2014;43(1):76-84 .

11. laboni A, Banez C, Lam R, Liu BA, Maki BE, Jones SA et al. The effect of depression on fear of falling in a falls prevention program. Am J Geriatr Psychiatry. 2015;23(10):1088-1097.

12. Barry E, Galvin R, Keogh C, Horgan F, Fahey T. Is the Timed Up and Go test a useful predictor of risk of falls in community dwelling older adults: a systematic review and meta- analysis. BMC Geriatr. 2014;14(1):2-14 .

13. Pimentel I, Schleicher ME. Comparação da mobilidade, força muscular e medo de cair em idosas caidoras e não caidoras. Rev Bras Geriatr e Gerontol. 2013;16(2):251257.

14. Lopes KT, Costa DF, Santos LF, Castro DP, Bastone BA. Prevalência do medo de cair em uma população idosos da comunidade e sua correlação com mobilidade, equilíbrio dinâmico, risco e histórico de quedas. Rev Bras Fisioter. 2009;13(3):223-229.

15. Podsiadlo D, Richardson S. The time up and go, a test of basic functional mobility for frail elderly persons. J Am Geriatr Soc. 1991;39(2):142-148.

16. Sun-Shil S, Durk-Hyun A. The Effect of Motor Dual-task Balance Training on Balance and Gait of Elderly Women. J Phys Ther Sci. 2014;26(3):359-361.

17. Plummer P, Zukowski LA, Guiliani C, Hall AM, Zurakowsk D. Effects of physical exercise interventions on gait-related dual-Task interference in older adults: A systematic review and meta-analysis. Gerontology. 2015;62(1):94117.

18. Cruz-Ferreira A, Fernandes J, Laranjo L, Bernardo LM, Silva A. A systematic review of the effects of pilates method of exercise in healthy people. Arch Phys Med Rehabil. 2011;92(12):2071-2081.

19. Barker AL, Bird ML, Talevski J. Effect of pilates exercise for improving balance in older adults: A systematic review with meta-analysis. Arch Phys Med Rehabil. 2015;96(4).

20. Francisco CDO, Fagundes ADA, Gorges B. Effects of Pilates method in elderly people: Systematic review of randomized controlled trials rewiew: Literature Review. J Bodyw Mov Ther. 2015;19(3):500-508.
21. Engers PB, Rombaldi AJ, Portella EG, da Silva MC. Efeitos da prática do método Pilates em idosos: Uma revisão sistemática. Rev Bras Reumatol. 2016;56(4):352-365.

22. Nascimento MDM, Cristina E, Carvalho F. A percepção da qualidade de vida de septuagenárias praticantes do método pilates. CPAQV. 2016;8(3):1-9.

23. Cancela JM, de Oliveira IM, Rodriguez-Fuentes G Effects of Pilates method in physical fitness on older adults. A systematic review. Eur Rev Aging Phys Act. 2014;11(2):81-94

24. Bullo EV, Bergamin M, Gobbo S, Sieverdes JC, Zaccaria $M$, Neuenhaeusere $D$ et al. The effects of Pilates exercise training on physical fitness and wellbeing in the elderly: A systematic review for future exercise prescription. Prev Med (Baltim). 2015;75.

25. Malta M, Cardoso LO, Bastos FI, Magnanini MMF, da Silva CMFP. STROBE initiative: guidelines on reporting observational studies. Rev Saude Publica. 2010;44(3):559-565.

26. Almeida O. Mini mental state examination and the diagnosis of dementia in Brazil. Arq Neuropsiquiatr. 1998;56(3B):605-612.

27. WHO-World Health Organization. Obesity: preventing and managing the global epidemic: report of a WHO consultation on obesity. Geneva. 1995.

28. Camargos FFO, Dias RC, Dias JMD, Freira TF. Adaptação transcultural e avaliação das propriedades psicométricas da Falls Efficacy Scale - International em idosos brasileiros (FES-I-BRASIL). Rev Bras Fisioter. 2010;14(3):237243.

29. De Castro SM, Perracini MR, Ganança FF. Dynamic gait index - Brazilian version. Braz J Otorhinolaryngol. 2006;72(6):817-825

30. Nascimento MM, Coriolano Appell I. Appell Coriolano HJ Teste de equilíbrio corporal (TEC) para idosos independentes. Rev Port Cienc Desp. 2012;12(2):72-82.

\section{CORRESPONDÊNCIA}

Marcelo de Maio Nascimento Colegiado de Educação Física da

Universidade Federal do Vale do São Francisco (UNIVASF)

E-mail: marcelo.nascimento@univasf.edu.br 